\title{
NATURAL SELECTION AND MATING CONSTRAINTS WITH GENETIC ALGORITHMS
}

\author{
Dana Vrajitoru \\ Intelligent Systems Laboratory \\ Computer and Information Sciences \\ Indiana University South Bend \\ South Bend, IN 46634, USA \\ email: danav@cs.iusb.edu
}

\begin{abstract}
In this paper we are modeling a situation in an evolutionary population where a genetically inherited feature is part of a distribution of probabilities that gives it low expectations to be present in the next generation, as for example, recessive features. In many such cases observed in nature, such features seem to persist longer than predicted by the probabilistic odds. In our experiment we intend to demonstrate how the fitness-proportionate selection can have an influence on the persistence of such features. In this simulation we focus on gender differentiation and on mating constraints and we examine their evolution in a mixed population where they are genetically inherited.
\end{abstract}

\section{Key Words}

Genetic algorithms, gender separation, social simulation, genetic diversity, mating constraints.

\section{Introduction}

In this paper we introduce a simulation that aims to observe the evolution of certain features in a population evolving through several generations. We use the known model of genetic algorithms (GAs) $[8,5]$ enhanced with the feature we intend to observe. The focus of the paper is on the influence of the fitness-proportionate selection on the distribution of values of a particular individual characteristic in a population as it evolves through several generations. We compare this aspect with a theoretical model using a probabilistic calculation based uniquely on inheritance in the absence of natural selection.

The feature that constitutes the focus of our present research is the gender type, and it can be extended to other individual characteristics with little modification. We start with a population containing several gender types and observe its convergence to one or two mating schemes through several generations under the influence of natural selection.

The study of the sexual reproduction and of the mating schemes has been an interest of research in GAs and evolutionary computation [17, 11, 19, 7, 21, 14, 15], especially in relation to the development of communication systems and of biodiversity.
The phenomenon of natural selection of the living beings combined with the preservation of the genetic diversity are among the most powerful adaptation schemes by which many species have survived and evolved into more intelligent ones. In this context, sexual reproduction is the most important mechanism for insuring the genetic diversity of a population and increasing the potential for adaptation of a species to hostile environments. Many studies have demonstrated the importance of preserving the biodiversity $[13,9]$, some of them in particular for the genetic algorithms [16].

Sexual reproduction with completely separate male and female organisms provides several advantages in nature. The most obvious one is that by preserving the biodiversity it contributes to the strength of the species, to its resistance to diseases, and to its capacity to adapt quickly to novel conditions. It has been observed that, in environments free of viruses like the polar or desert regions, and where life is sparse, self-fertilizing organisms are more widespread than in well populated areas [6]. These authors relate their findings to the presence or absence of viruses in those regions and conclude that the existence of two gender types in a species provides it with better chances to adapt to new situations and harsher environments.

Some studies have also shown that sexual differentiation is instrumental in developing communication within a population. Gender differentiation has compelled the organisms to develop sophisticated mating schemes, which enhance the natural selection of the best individuals for reproduction [24]. Recruiting an individual of another gender for mating purposes requires among other things a complex communication system whose development may have contributed to evolving language and social structure, both of which are closely related to the intelligence of a species.

Other interesting research concerning gender approaches the problem of multi-optimization [1]. In this context, sexual selection can be regarded as a cooperation between two individuals or even two genetic operators to solve a symbiotic problem [10].

In this paper we introduce a model where several reproductive modes coexist and their survival depends on the natural selection in a simulation using genetic algorithms. We can classify these organisms in three major categories 
according to their mating mechanism.

The first type, called self-fertilizing, is manifest in many plants and consists of individuals that present both male and female features simultaneously, thus being capable of producing offspring without any exterior contribution. The offspring are practically genetic clones of the parent.

The second category contains organisms that present both male and female organs, but that have developed a mechanism to prevent the self-fertilization. For example, some plants develop the male and female organs at different moments in time, while some hermaphrodite species of fish can change their sex at particular stages of their life.

The third category includes all the other multicellular species, in particular the majority of the animals, for which each individual develops only one of the two possible reproductive organs and thus can only mate with individuals of a different gender. This represents in general about half of the population, although for some species an imbalance in the distribution of the two genders can be observed.

The experiment presented in this paper analyzes the influence of the natural selection on the evolution of the reproduction mode. For this, we start with a population with a balanced distribution of three reproduction modes and evolve the gene representing this feature along with the rest of the genetic code. This experiment considers a mixed population where several reproduction types coexist. Even if such cases are not currently observed in nature, we can suppose that when sexual differentiation occurred initially in unicellular organisms, some period of coexistence might have been possible. It can also represent in a symbolic way social gender choices such as heterosexual, bisexual, and so on. We compare the evolution of such population with a theoretical model that ignores the natural selection. This part of the paper is a continuation of [21].

The remainder of the paper is structured as follows. Section 2 presents the general outline of our gender evolution model. Section 3 presents a prediction of the behavior of our model in a context without natural selection. Section 4 follows up with a set of simulations in which the natural selection is present, followed by some conclusions.

\section{Modeling a Multi-Gender Population}

In this section we present a simulation model in which an evolutionary population in which individuals of several reproduction modes coexist, namely asexual allowing but not restricted to self-fertilizing $(\mathrm{SF})$, hermaphrodite $(\mathrm{H})$ where the two parents for crossover must be different, male (M), and female $(\mathrm{F})$. This feature is inherited by the children in the crossover process and evolves through several generations. In our experiments, the population always converges to one $(\mathrm{SF}, \mathrm{H})$ or two $(\mathrm{M} / \mathrm{F})$ gender types after a number of generations. We are interested in the probability of convergence to each type of reproduction with and without natural selection.

\subsection{The System}

We are interested in the evolution of a heterogeneous population from a gender point of view under the influence of the natural selection. We can see the population as a system with the individual chromosomes being the components of the system. The behavior of these chromosomes is not in fact the main issue in our simulation, but the actual composition of the population in terms of gender types. Thus, we will consider that our system as composed of four variables, each of them representing the number of chromosomes in the current state (or generation).

In our model, we have four types of individuals: SF, $\mathrm{H}, \mathrm{M}$, and $\mathrm{F}$. If we denote by $n_{S F}, n_{H}, n_{M}$, and $n_{F}$, the number of self-fertilizing, hermaphrodite, male, and female individuals respectively in a given generation, we can represent the state of the system as the vector

$$
\left\langle n_{S F}, n_{H}, n_{M}, n_{F}\right\rangle
$$

We would like to see the evolution of this system through several generations under inheritance conditions.

\subsection{State Transition}

For a complete model, we need to define the initial state of the system and the transition from each state to the next. The evolution of our system represents a Markov chain where each new state is obtained from the previous one by operations specific to the GAs.

Initial state. The gender of the individual is assigned at random in the initial population, following a fair distribution of probabilities such that we have an approximately equal representation of each gender, and is also inherited by the children from their parents. Thus, the reproduction mode specific to the individuals selected for reproduction will be inherited by the new generation.

Transition. Since the states of the system represent distinct generations in the execution of the GA, the transition is defined by the mating restrictions specific to each gender type and by the genetic operations used to build the new generation from the current one. For the purpose of our simulation, we must define precisely the mating and inheritance mechanism for each gender type, which determines the gender composition in the next state.

A self-fertilizing individual can mate with any individual, including itself. The hermaphrodite individuals can mate with any individual other than themselves. The males and females can mate with an individual of the opposite sex or with any of the two other kinds (SF and H). To prevent deadlocks in the case where the population is entirely male or entirely female, we allow for spontaneous gender change if the mating process fails for a given number of attempts.

The offspring inherit the gender type of their parents randomly, with an equal probability for the gender of each parent, except for the male/female types. A parent of male/female gender can spawn offspring of either male or 
female type with no bias towards one of them. Thus, the child of a hermaphrodite and a male could be a female with a probability of $25 \%$.

The factor that we want to examine in our simulation is the influence of the fitness-proportionate selection over the evolution of the system as we defined it in the previous subsection. For this purpose, we will consider two types of transitions, with and without fitness. In the first case, the transition from one state to the next is uniquely determined by the expected number of individuals in the new generation based on the current situation or by random choices of parents. In the fitness-based evaluation, the transition follows the rules of evolution within the genetic algorithms, where the fitness function determines the probability of survival of each individual from the current generation. Thus, the number of individuals of each gender type in the next generation, representing the new state of the system, will be determined both by the probabilities associated with the mating mechanism and by the fitness-proportionate selection.

\subsection{Model Validation and Reusability}

To validate our model we will compare the theoretical prediction with the experimental results done with a fitnessproportionate selection. The difference between the two types of system evolution will emphasize the influence of the fitness-proportionate selection on the convergence of the system.

This simulation is specific to gender types, but it can be extended to any features of the individuals in a population that are genetically inherited, going from physical attributes like height and hair color, to genetically transmitted diseases. We also intend to show using our model how recessive features can survive in a population and even become predominant in some situations.

In terms of system reusability, the gender type can be incorporated in the general execution of the genetic algorithms to enhance particular aspects, like preserving the biodiversity, which has been proved to improve the general performance of the population.

\section{State Transition without Natural Selection}

In this section we introduce two experiments designed to predict the expected composition of the population in several generations without the natural selection.

\subsection{Expected Population Composition}

Let us denote by $n_{0 M}, n_{0 F}, n_{0 S F}$, and $n_{0 H}$ the number of male, female, self-fertilizing, and hermaphrodite individuals respectively in the current generation or state of the system. Let us compute the expected number of occurrences of each gender type in the next generation, denoted by $n_{1 M}, n_{1 F}, n_{1 S F}$, and $n_{1 H}$, considering the mat- ing scheme we have described without the intervention of the natural selection. Let $n=n_{0 M}+n_{0 F}+n_{0 S F}+n_{0 H}$ be the size of the population. To simplify the computation, let $n_{0 M F}$ and $n_{1 M F}$ be the number of individuals that are either males or females in the old and new generation respectively, $n_{i M F}=n_{i M}+n_{i F}, i=0,1$.

For the male/female individuals, their type can be found in the next generation in either of these situations: if a male or female are chosen as the first parent, or if an individual of any other type is chosen as the first parent and it is matched by a male-female individual. The probability that one chosen individual is a male or female is equal to $n_{0 M F} / n$, the number of such individuals divided by the size of the population.

To simplify the equation, let us consider that the number of males and of females is about the same, and let it be $m_{0}=n_{0 M F} / 2$. If we also note that $n_{0 H}+n_{0 S F}=$ $n-2 m_{0}$, then we have that

$$
\begin{gathered}
E\left(n_{1 M F}\right)= \\
=m_{0}\left(\frac{2 m_{0}}{n-m_{0}}+\frac{n-2 m_{0}}{n-m_{0}}+\frac{n_{0 H}}{n-1}+\frac{n_{0 S F}}{n}\right)= \\
=m_{0}\left(\frac{n}{n-m_{0}}+\frac{n-2 m_{0}}{n-1}+\frac{n_{0 S F}}{n(n-1)}\right)= \\
=m_{0}\left(\frac{2 n^{2}+2 m_{0}^{2}-3 n m_{0}^{2}-n}{(n-1)\left(n-m_{0}\right)}-\frac{n_{0 S F}}{n(n-1)}\right)
\end{gathered}
$$

By ignoring the last term in Equation 1 as a small negative quantity, let us see how the expected number of male and female individuals compares to the previous one, $2 m_{0}$. We will prove that the expected number in the new generation is lower than their number in the previous one.

$$
E\left(n_{1 M F}\right) \leq 2 m_{0} \Leftrightarrow\left(m_{0}-1\right)\left(2 m_{0}-n\right) \leq 0
$$

In Equation 2, we can assume that $m_{0} \geq 1$, otherwise $n_{1 M F}=0$. What remains to verify is that $2 m_{0} \leq n$. If we remember that we have made the assumption that $n_{0 M F}=2 m_{0}$, this means that if either the number of hermaphrodite or of self-fertilizing individuals in the population is not 0 , then the population of male/female individuals will decrease from each generation to the next.

We can find a hermaphrodite in the new generation if a hermaphrodite was chosen as a first parent, or if the second parent is a hermaphrodite and not identical to the first one. The probability of the second case requires a complex computation because if the first parent is a male, a hermaphrodite individual will be chosen from the entire population except for the male individuals, which increases their chances. Their expected number is

To simplify Equation 3 we will make the same assumption as for Equation 1. Then we can rewrite Equation 3 as shown in Equation 4.

$$
E\left(n_{1 H}\right)=\frac{n}{2}\left(\frac{n_{0 H}}{n}+\frac{n_{0 S F}}{n} \frac{n_{0 H}}{n}+\frac{n_{0 M}}{n} \frac{n_{0 H}}{n-n_{0 M}}+\right.
$$




$$
\begin{gathered}
\left.+\frac{n_{0 F}}{n} \frac{n_{0 H}}{n-n_{0 F}}+\frac{n_{0 H}}{n} \frac{n_{0 H}-1}{n-1}\right) \\
E\left(n_{1 H}\right)=\frac{n_{0 H}}{2}\left(1+\frac{n-2 m_{0}}{n-1}+\frac{2 m_{0}}{n-m_{0}}-\right. \\
\left.-\frac{n+n_{0 S F}}{n(n-1)}\right)
\end{gathered}
$$

Considering that the last factor in the previous equation is a negligible quantity, if $m_{0} \geq 1$, we can say that

$$
E\left(n_{1 H}\right) \geq \frac{n_{0 H}}{2}\left(1+\frac{n}{n-1}\right) \geq n_{0 H}
$$

We can interpret Equations 4 and 5 to indicate that as long as the number of male/female individuals is not negligible, the number of self-fertilizing individuals is expected to increase. Then if the number of male/female individuals is close to 0 , the expected number of hermaphrodite individuals will slowly decrease in favor of the self-fertilizing ones.

The number of self-fertilizing individuals is computed similarly to the hermaphrodite individuals, except that the parents can be the same individual. The equation is:

$$
\begin{aligned}
E\left(n_{1 S F}\right)= & \frac{n}{2}\left(\frac{n_{0 S F}}{n}+\frac{n_{0 S F}}{n} \frac{n_{0 S F}}{n}+\frac{n_{0 M}}{n} \frac{n_{0 S F}}{n-n_{0 M}}+\right. \\
& \left.+\frac{n_{0 F}}{n} \frac{n_{0 S F}}{n-n_{0 F}}+\frac{n_{0 H}}{n} \frac{n_{0 S F}}{n-1}\right)
\end{aligned}
$$

By making the same assumption as for Equation 3, we can rewrite Equation 6 the following way:

$$
\begin{gathered}
E\left(n_{1 S F}\right)=\frac{n_{0 S F}}{2}\left(1+\frac{n_{0 S F}}{n}+\frac{n_{0 H}}{n-1}+\frac{2 m_{0}}{n-m_{0}}\right)= \\
=n_{0 S F}+\frac{n_{0 S F}}{2}\left(\frac{m_{0}^{2}}{n\left(n-m_{0}\right)}+\frac{n_{0 H}}{n(n-1)}\right)
\end{gathered}
$$

Equation 7 tells us that as long as the population is not entirely composed of self-fertilizing individuals, their number is expected to increase. It is also notable that the increment of the number of self-fertilizing individuals depends more on the male/female individuals than on the hermaphrodite ones. This means that as long as the population contains some male/female individuals, the number of self-fertilizing individuals will grow at a fast rate. When the population contains only hermaphrodite and self-fertilizing individuals, their numbers will evolve a lot slower.

Using Equations 1 to 7 recursively, we predicted the evolution of the system in the absence of natural selection. Thus, this part of the experiment is deterministic. We plotted the expected numbers of individuals of each of the four types for a population size of 50 over 500 generations, starting from an equal number of individuals of each type (12.5). Figure 1 shows the results of this simulation.

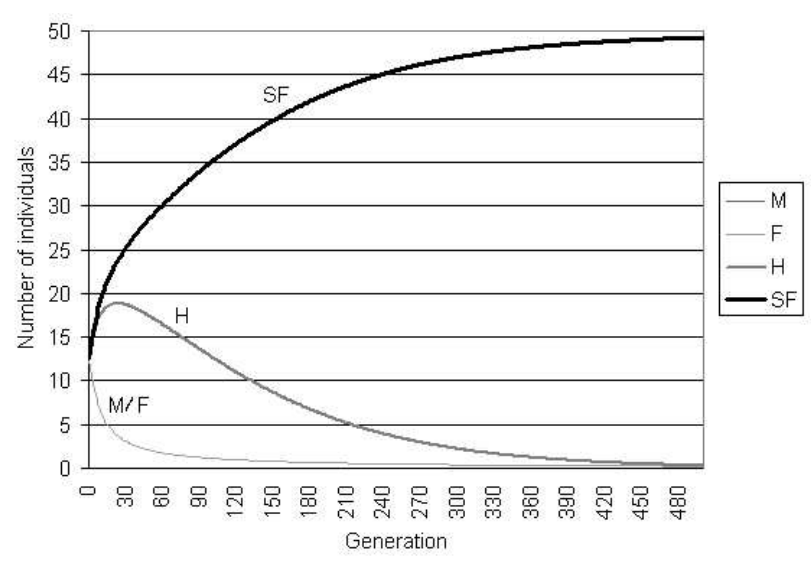

Figure 1. Theoretical evolution of the number of individuals of each gender type for a population of size 50 in 500 generations

In this figure we can see that the number of self-fertilizing individuals is continuously growing until it almost dominates the population. The number of hermaphrodite individuals is growing while the number of male/female individuals is still large enough, then it starts to decrease, but at a slower rate than the male/female individuals. The number of male/female individuals becomes less than 1 in generation number 117, while the number of hermaphrodites becomes less than 1 only in generation number 380 . The experiences presented in the next section employing a genetic algorithm have shown that the population converges to one gender type a lot faster than in the theoretical case, in general after less than 50 generations.

\subsection{Constant Fitness Simulation}

To validate the theoretical calculation done in the previous subsection, we ran an experiment with the GA in which the fitness function has a constant value. Thus, this simulation is non-deterministic, but is also conducted without the influence of the natural selection. The individuals from the current generation are selected for reproduction in an unbiased random manner.

In this experiment we start with a population composed of equal numbers of each gender type and run the GA for 500 generations. We performed 50 runs of the GA with an initial population size of 50. The gender type is inherited by the children from the parents as we described in Section 2. The only feature that has meaning here is the gender type as we are not trying to solve any other concrete problem and the fitness is constant. We look at the composition of the population at the end of the evolutionary process. This gives us an idea of what the expected gender evolution is if we only consider the probabilities derived from the mating constraints.

Figure 2 shows the result of this simulation in the first 215 generations, since the population had completely con- 
verged at that point in all of the 50 trials. The generation number at which we observed complete convergence in all cases is 212 , but the generation number at which the male/female individuals disappeared is 109 .

If we compare this figure with Figure 1, we notice that in this second case, the convergence curve is less smooth but the general outline of the evolution of the system is similar. The difference between the two simulations is that Figure 1 presents a theoretical computation of the sequence of expected states of the system based on the probabilities introduced in the previous subsection, while Figure 2 presents a practical experiment. In the latter simulation individuals are selected randomly regardless of their fitness, but the mating constraints are the same as in the case of any population in the context of the GAs.

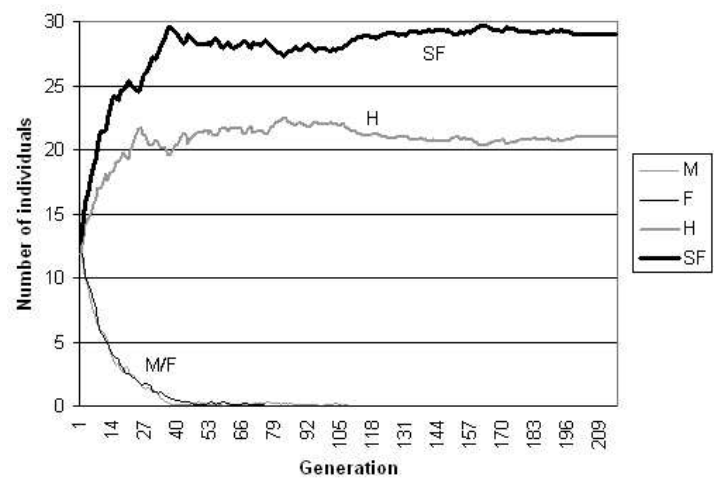

Figure 2. Simulation of gender evolution with a population size of 50 in 500 generations ( 215 shown), constant fitness

These two analysis completed without considering the influence of the natural selection represents the baseline for our experiment. In the next section the fitnessproportionate selection will choose parents of high fitness with a greater probability and the expected occurrence of their gender type in the new generation will be higher.

\section{State Evolution with Natural Selection}

In the experiment presented in this section, we observe the evolution of a population in the context of a class genetic algorithm where the chromosomes or individuals are augmented with an additional gene for the gender type. The gender is inherited in the evolution process as described in Section 2.

For a better reliability of our results we used several fitness functions with various landscapes.

\subsection{Test Functions}

We have chosen three classes of problems to test our model: a set of ten standard test functions, several deceptive functions, and one NP-complete problem. Each class of problems presents a special challenge for the GAs, and a combi- nation of them can give us a better idea of the mechanisms in the model that we try to simulate.

Standard functions set. We started our experiments with a set of ten standard functions used in many cases to test GAs [25]. The goal is to find values of the variables used by the functions representing a minimum for each function. The full equations for these problems can be found in [23]. The size of an individual is in general ten times the number of variables involved in the description of its function, meaning that ten genes are used to encode each variable.

Deceptive problems. This class of problems is based on the phenomenon of deception [4] and contains problems that are known to be difficult for GAs. For this reason, they are a frequent choice as test functions in the study of GAs [12]. Their difficulty comes from the fact that the optimal individual is isolated from other individuals of high performance, and there are one or more suboptimal individuals that are easier to reach by hill-climbing.

We chose eight deception problems that consist in concatenating a given number of 3-bit functions, described in detail in [23]. For these problems, the optimal individual is represented by a string of 3 bits whose closest neighbors display the lowest performance. We have conducted our experiments with individuals composed of 1003 -bit strings, making the fitness of the optimal individual 3000.

Hamiltonian circuit $(H C)$. Given an oriented graph, does there exist a circuit that passes once and only once through each vertex? This problem is NP-complete.

We performed our experiments with ten HC problems concerning graphs of 9 to 150 vertices and up to 3000 edges. The direct representation of a $\mathrm{HC}$ problem for the GAs is difficult. De Jong and Spears [3] suggest transforming the $\mathrm{HC}$ instances into instances of the satisfiability (SAT) problem, described bellow, whose genetic representation is easier.

SAT (Boolean satisfiability). Given a Boolean expression depending on some variables, does there exist an assignment to those variables such that the value of the expression becomes true?

A detailed description of the reduction of a $\mathrm{HC}$ instance into a SAT instance can be found in [20,23]. For any given graph, a Boolean variable corresponds to each edge, and is given the true value if the edge belongs to the circuit. The SAT expression represents the fact that, for each vertex, one and only one of the entering edges and of the exiting edges must belong to the circuit. This translates into an 'xor' clause binding all the edges entering each vertex and a similar one for the edges exiting each vertex. The entire expression is built by combining all the clauses with the 'and' operator.

The genetic representation of SAT is straightforward. Each variable is mapped onto a binary gene, where the 0 / 1 values can be interpreted as false/true. To evaluate an expression to more than true or false, we used fuzzy logic measures [3]. Specifically, the 'and' operation is evaluated to the average of the terms, while the 'or' operation returns 
the maximum of the terms.

GA Settings. We have used the combined balanced crossover operator [22]. This operator utilizes four variations of crossover to build every new generation: 1-point [8], 2-point [2], uniform [18] with a swap probability of 0.5 , and dissociated [20]. For each operation, one of the four crossover forms is chosen randomly, each of them having an equal probability to be selected. The probability of mutation varies from 0 to 0.01 .

\subsection{Experimental Results}

As described in Section 3, the initial population has a balanced composition in terms of gender types. We are interested in the composition of the population after running the GA for 500 generations.

We performed 150 runs of the GA for each problem with a different initial population. 50 of the trials were with a mutation rate of 0 (no mutation), 50 of them with a mutation rate of 0.0005 , and 50 of them with a mutation rate of 0.01 . The crossover rate is equal to 1 in all the cases. We performed each experiment with an initial population size of 50 .

The results of our experiments with the three types of problems are shown Tables 1 and 2. We display the percentage of simulations for which the population has converged to each of the gender types, for each class of problems, and for population sizes of 50 and 100 respectively. These results are compared with the convergence number for each gender type in the simulations presented in Figures 1 and 2, denoted in the table by constant fitness. In our experiments, the population has always converged to one particular gender type in 500 generations, and often a lot sooner than that.

We note that although the most likely convergence type is SF in all cases, with and without fitness, the actual percentages of convergence to each type are quite different. Thus, the number of populations composed of male/female is not equal to the expected number (0). Also, the number of hermaphrodite individuals is higher than the expected one. These discrepancies are due to the influence of the fitness-proportionate selection. For the larger populations, the results are closer to the probabilistic expectations and this is most likely due to the probabilistic law of big numbers.

From these experiments we can deduce that the phenomenon of natural selection determines the choice of the reproduction mode in the new generation and this is why there is a difference between the theoretical simulation presented in Section 3 and the experimental results. In a situation where the gender types represent social choices and not biological genders, these results can be interpreted as the fact that the gender choices of the dominant individuals in the population or those promoted by institutions of influence (church, state) will have an influence on the individual choices.
Table 1. Gender convergence in 500 generations, population size of 50

\begin{tabular}{lllll} 
& Fitness & $\mathrm{M} / \mathrm{F}$ & $\mathrm{H}$ & $\mathrm{S}-\mathrm{F}$ \\
\hline \multirow{4}{*}{$p_{m}=0$} & & & & \\
& Const & 0 & $42 \%$ & $58 \%$ \\
\hline & Standard & $1.00 \%$ & $45.80 \%$ & $53.20 \%$ \\
& Deception & $0.50 \%$ & $47.00 \%$ & $52.50 \%$ \\
& HC & $0.57 \%$ & $46.29 \%$ & $53.14 \%$ \\
& Average & $0.69 \%$ & $46.36 \%$ & $52.95 \%$ \\
\hline \multirow{5}{*}{$p_{m}=0.0005$} & Standard & $0.00 \%$ & $42.67 \%$ & $57.33 \%$ \\
& Deception & $0.00 \%$ & $46.34 \%$ & $53.66 \%$ \\
& HC & $0.00 \%$ & $47.90 \%$ & $52.10 \%$ \\
& Average & $0.00 \%$ & $45.64 \%$ & $54.36 \%$ \\
\hline \multirow{5}{*}{$p_{m}=0.01$} & Standard & $1.00 \%$ & $45.80 \%$ & $53.20 \%$ \\
& Deception & $0.50 \%$ & $48.00 \%$ & $51.50 \%$ \\
& HC & $0.48 \%$ & $46.38 \%$ & $53.14 \%$ \\
& Average & $0.66 \%$ & $46.73 \%$ & $52.61 \%$ \\
\hline \multirow{5}{*}{ Global } & Average & $0.68 \%$ & $46.54 \%$ & $52.78 \%$
\end{tabular}

Table 2. Gender convergence in 500 generations, population size of 100

\begin{tabular}{lllll} 
& Fitness & $\mathrm{M} / \mathrm{F}$ & $\mathrm{H}$ & $\mathrm{S}-\mathrm{F}$ \\
\hline & & & & \\
& Const & $0 \%$ & $40 \%$ & $60 \%$ \\
\hline \multirow{5}{*}{$p_{m}=0$} & & & & \\
& Standard & $0.20 \%$ & $48.19 \%$ & $51.61 \%$ \\
& Deception & $0.00 \%$ & $46.02 \%$ & $53.98 \%$ \\
& HC & $0.00 \%$ & $41.65 \%$ & $58.35 \%$ \\
& Average & $0.07 \%$ & $45.29 \%$ & $54.65 \%$ \\
\hline \multirow{5}{*}{$p_{m}=0.0005$} & & & & \\
& Standard & $0.20 \%$ & $46.37 \%$ & $53.41 \%$ \\
& Deception & $0.00 \%$ & $37.73 \%$ & $62.27 \%$ \\
& HC & $0.00 \%$ & $44.97 \%$ & $55.03 \%$ \\
& Average & $0.07 \%$ & $43.02 \%$ & $56.9 \%$ \\
\hline \multirow{5}{*}{$p_{m}=0.01$} & Standard & $0.20 \%$ & $47.15 \%$ & $52.65 \%$ \\
& Deception & $0.00 \%$ & $42.00 \%$ & $58.00 \%$ \\
& HC & $0.00 \%$ & $43.86 \%$ & $56.14 \%$ \\
& Average & $0.07 \%$ & $44.34 \%$ & $55.59 \%$ \\
\hline \multirow{2}{*}{ Global } & Average & $0.07 \%$ & $44.22 \%$ & $55.71 \%$
\end{tabular}




\section{Conclusions}

In this paper we introduced a model simulating a variety of mating schemes. The main goal of our paper was to explore the influence of the natural selection on the evolution of some individual features such as the gender. We were also interested in the role of social factors such as the size of the population and mating restrictions on the performance achieved by the evolving population in terms of fitness.

The first presented experiment consisted in a system where several reproduction modes including hermaphrodite and with sexual differentiation compete for dominance in the same population. A calculation of expected state transition followed by a deterministic simulation showed that in the absence of natural selection, in a short term the sexual differentiation feature disappears, and in the longer term, the hermaphrodite population is also eliminated in favor of the simple asexual reproduction.

Our experiments show the features characterizing the individuals of high fitness tend to become dominant in the population and can override the probabilistic predictions, as seen in Sections 3 and 4. The natural selection being the major difference between these simulations, we can entail that this is the factor allowing features with low probabilistic expectations to survive and even become dominant in the population. This model can be extended to genetically recessive features of the individuals.

\section{References}

[1] R. Allenson. Genetic algorithms with gender for multi-function optimisation. Technical Report EPCCSS92-01, Edinburgh Parallel Computing Centre, Edinburgh, Scotland, 1992.

[2] K. De Jong. An Analysis of the Behaviour of a Class of Genetic Adaptive Systems. PhD thesis, University of Michigan, 1975.

[3] K. De Jong and M. Spears. Using genetic algorithms to solve NP-complete problems. In Proceedings of the International Conference on Genetic Algorithms, pages 124-132, Fairfax (VA), 1989. George Mason University.

[4] K. Deb and D. E. Goldberg. Sufficient conditions for arbitrary binary functions. Annals of Mathematics and Artificial Intelligence, 10:385-408, 1994.

[5] D. E. Goldberg. Genetic Algorithms in Search, Optimization, and Machine Learning. Addison-Wesley, Reading (MA), 1989.

[6] W.D. Hamilton, R. Axelrod, and R. Tanese. Sexual reproduction as an adaptation to resist parasites. Proceedings of the National Academy of Sciences, 87:3566-3573, May 1990.
[7] C.K. Hemelrijk. Effects of cohesiveness on intersexual dominance relationships and spatial structure among group-living virtual entities. In Proceeding of the European Conference on Artificial Life V, pages 524-534. Springer Verlag, 1999.

[8] J. H. Holland. Adaptation in Natural and Artificial Systems. University of Michigan Press, Ann Arbor, 1975.

[9] N.D. Martinez. Biodiversity: A Biology of Numbers and Difference, chapter Defining and measuring functional aspects of biodiversity, pages 114-148. Blackwell Scientific, Oxford, UK, 1996.

[10] J. Sanchez-Velazco and J.A. Bullinaria. Sexual selection with competitive/co-operative operators for genetic algorithms. In IASTED International Conference on Neural Networks and Computational Intelligence (NCI 2003). ACTA Press, 2003.

[11] G.F. Miller and P.M. Todd. The role of mate choice in biocomputation: Sexual selection as a process of search, optimization, and diversification. In W. Banzhaf and F.H. Eeckman, editors, Evolution and biocomputation: Computational models of evolution, pages 169-204, Berlin, 1995. Springer-Verlag.

[12] C. K. Mohan. Selective crossover: Towards fitter offspring. In Proceedings of the Symposium on Applied Computing (SAC'98), Atlanta (GA), 1998.

[13] S. Naeem, L.J. Thompson, S.P. Lawlor, J.H. Lawton, and R.M. Woodfin. Declining biodiversity can alter the performance of ecosystems. Nature, (386):734737, 1994.

[14] J. Noble. Sexual signalling in an artificial population: When does the handicap principle work? In D. Floreano, F. Mondada, and J.-D. Nicoud, editors, Proceeding of the European Conference on Artificial Life V, pages 644-653. Springer Verlag, 1999.

[15] J. Rejeb and M. AbuElhaija. New gender genetic algorithm for solving graph partitioning problems. In 43rd IEEE Midwest Symposium on Circuits and Systems, volume 1, pages 444-446. IEEE, 2000.

[16] J. D. Schaffer and L. Eshelman. On crossover as an evolutionary viable startegy. In R. Belew and L. Booker, editors, Proceedings of the Fourth International Conference on Genetic Algorithms, pages 6168, San Mateo (CA), 1991. Morgan Kaufmann Publishers.

[17] B.H. Sumida, A.I. Huston, J.M. McNamara, and W.D. Hamilton. Genetic algorithms and evolution. Journal of Theoretical Biology, (147):59-84, 1990.

[18] G. Syswerda. Uniform crossover in genetic algorithms. In J. D. Schaffer, editor, Proceedings of the 
International Conference on Genetic Algorithms, San Mateo (CA), 1989. Morgan Kaufmann Publishers.

[19] J. Ventrella. Sexual swimmers: Emergent morphology and locomotion without a fitness function. In Proceedings of the Fourth International Conference on Simulation of Adaptive Behavior, pages 484-496. Bradford Books, 1996.

[20] D. Vrajitoru. Genetic programming operators applied to genetic algorithms. In Proceedings of the Genetic and Evolutionary Computation Conference, pages 686-693, Orlando (FL), 1999. Morgan Kaufmann Publishers.

[21] D. Vrajitoru. Simulating gender separation with genetic algorithms. In Proceedings of the Genetic and Evolutionary Computation Conference, pages 634641. Morgan Kaufmann Publishers, 2002.

[22] D. Vrajitoru. Intra and extra-generation schemes for combining crossover operators. In The Midwest Artificial Intelligence and Cognitive Science Conference, pages 86-91, Roosevelt University, IL, 2004.

[23] D. Vrajitoru. Simulating gender separation and mating constraints for genetic algorithms. Technical Report TR-20050520-1, Indiana University South Bend, 2005.

[24] G.M. Werner and P.M. Todd. Too many love songs: Sexual selection and the evolution of communication. In P. Husbands and I. Harvey, editors, The Fourth European Conference on Artificial Life, Cambridge (MA), 1997. MIT Press/Bradford Books.

[25] D. Whitley, K. Mathias, S. Rana, and J. Dzubera. Evaluating evolutionary algorithms. Artificial Intelligence, 85:245-276, 1996. 